\title{
Feeling happy enhances early spatial encoding of peripheral information automatically: electrophysiological time-course and neural sources
}

\section{Running title:}

\section{Happy mood broadens attention automatically}

Naomi Vanlessen, Valentina Rossi, Rudi De Raedt \& Gilles Pourtois

Psychopathology and Affective Neuroscience Laboratory, Department of Experimental Clinical \& Health Psychology, Ghent University, Ghent, Belgium

Correspondence :

Gilles Pourtois

Department of Experimental-Clinical and Health Psychology

Ghent University

Henri Dunantlaan 2

9000 Gent, Belgium

Phone: +3292649144

Email: gilles.pourtois@ugent.be 


\section{ABSTRACT}

Previous research showed that positive mood may broaden attention, although it remains unclear whether this effect has a perceptual or post-perceptual locus. In this study, we addressed this question using high density event-related potentials (ERP) methods. We randomly assigned participants to a positive or neutral mood condition. Then, they performed a demanding oddball task at fixation (primary task ensuring fixation) and a localization task of peripheral stimuli shown at three positions in the upper visual field (secondary task) concurrently. While positive mood did not influence behavioral performance for the primary task, it did facilitate stimulus localization on the secondary task. At the electrophysiological level, we found that the amplitude of the $\mathrm{C} 1$ component (reflecting an early retinotopic encoding of the stimulus in V1) was enhanced in the positive compared to the neutral mood group. Importantly, this effect appeared to be largely automatic, because it occurred regardless of the task relevance of the peripheral stimulus, and prior to top-down gain control effects seen at the level of the subsequent P1 component. This early effect was also observed irrespective of a change of the target-related P300 component (primary task) by positive mood. These results suggest that positive mood can automatically boost the spatial encoding of peripheral stimuli early on following stimulus onset. This effect can eventually underlie the broadening of spatial attention which has been associated with this specific mood state.

Keywords: positive emotion, attention, ERP, C1, V1, P300 


\section{INTRODUCTION}

\section{The broaden and build effects of positive emotions}

The importance of positive emotions in psychological wellbeing has increasingly gained researchers' interest since Fredrickson published her influential broaden-and-build theory (Fredrickson, 2001, 2004). At the heart of this theory lies the idea that positive and negative emotions exert opposite influences on cognitive functions: whereas negative mood would trigger a narrowing of the attentional scope and behavioral repertoire, positive mood on the other hand would fuel broader thought-action tendencies and expand the attentional focus (Fredrickson \& Branigan, 2005). Evidence supporting this theory comes from Derryberry and Tucker (1994), who found an association between positive mood and a larger attentional scope, and from Isen and her colleagues, who showed that positive affect enables a flexible (Isen \& Daubman, 1984), creative (Isen, Daubman, \& Nowicki, 1987), integrative (Isen, Rosenzweig, \& Young, 1991) and open (Estrada, Isen, \& Young, 1997) way of processing information and seeking solutions (see Isen, 2000 for a review).

An expansion of the attentional focus might be crucial to the benefits of positive mood for higher order cognitive functioning, and different paradigms have been set up to reveal such an effect, including flanker and other interference tasks. While some studies found evidence for a broadening effect of positive mood (Moriya \& Nittono, 2011; Rowe, Hirsh, \& Anderson, 2007), others failed to replicate this effect (Bruyneel, et al., 2013; Finucane, Whiteman, \& Power, 2010; Huntsinger, Clore, \& Bar-Anan, 2010; Martin \& Kerns, 2011). These discrepant results might stem from the fact that these earlier studies primarily used interference tasks, which are not pure measures of spatial attention, but instead rely more heavily on executive functions and cognitive control (Botvinick, Braver, Barch, Carter, \& Cohen, 2001). Because conflict and competition among multiple responses are induced by these interference tasks, it is likely that the effects reported in some of these earlier studies 
concern post-perceptual stages of processing rather than a genuine broadening of the attentional focus early on following stimulus onset. Hence, using these tasks, it remains unclear whether positive mood influences early or late stages of information processing during attention selection.

\section{Costs and benefits of a broadening of attention under positive mood}

To overcome these limitations, we previously validated a task enabling us to titrate effects of positive mood on early stages of visual processing and attention selection, both at the EEG and behavioral levels (Vanlessen, Rossi, De Raedt, \& Pourtois, 2013). In this earlier study, participants were randomly assigned to either a neutral or a positive mood induction condition that consisted of a standard guided imagery procedure (Holmes, 2006; Holmes, Coughtrey, \& Connor, 2008). Next, they performed a demanding oddball detection task in the center of the screen, used to ensure fixation throughout the experimental session. Orthogonally, distractors (i.e., unattended textures) were briefly flashed at different eccentricities in the upper visual field at an unpredictable time and location relative to these central stimuli. These task parameters were therefore suited to study two major components of attention control concurrently: top-down attention selection (indexed by the processing of the central stimuli) and bottom-up attention capture (indexed by the covert or implicit processing of the peripheral distractors; Corbetta \& Shulman, 2002; Schwartz, et al., 2005).

At the EEG level, these two processes were formally operationalized by amplitude changes at the level of the P300 and C1 components, respectively. Oddball targets elicited a conspicuous P300 component relative to standards, in line with previous results (Rossi \& Pourtois, 2012). However, this effect was not influenced by positive mood. The $\mathrm{C} 1$ elicited by the peripheral distractors varied in amplitude with their actual spatial position, being larger for textures shown close to fixation compared to further away in the upper visual field (Clark, 
Fan, \& Hillyard, 1995). Remarkably, this near-far gradient effect was influenced by positive mood, indicated by a less sharp decrease of the $\mathrm{C} 1$ amplitude with increasing eccentricity for participants in the positive compared to the neutral mood group. Given that the $\mathrm{C} 1$ corresponds to the earliest sweep of activation in the primary visual cortex following stimulus onset (Jeffreys \& Axford, 1972; Rauss, Schwartz, \& Pourtois, 2011), we interpreted these results as reflecting an early influence of positive mood on the processing of peripheral distractors (bottom-up component of attention). Moreover, the direction of this ERP effect was compatible with a broadening of attention under positive mood, as if in this specific mood state attention was readily allocated to peripheral distractors falling far away relative to fixation.

At the behavioral level, we reasoned that this neurophysiological effect would not necessarily translate as a measurable advantage in the processing of the content of these stimuli as a function of positive mood. Earlier studies investigating the effects of a broadened attentional scope showed a trade-off between the size of the attentional focus and the spatial resolution within that focus (Castiello \& Umilta, 1990; Eriksen \& Yeh, 1985; Ivry \& Robertson, 1998), with corresponding effects at the neural level (Muller, Bartelt, Donner, Villringer, \& Brandt, 2003). Thus, when attentional resources are spread over a larger portion of the visual field, this gain in spatial attention is somehow counteracted by a loss regarding the processing of the details and local information. In line with this prediction, we found in our previous study (Vanlessen, et al., 2013) that participants in the positive mood group showed a drop in accuracy for processing the content (i.e., local elements) of these textures shown in the upper visual field, compared to the participants in the neutral mood group. However, this behavioral effect was evidenced only in a control experiment where no EEG was recorded concurrently (Vanlessen, et al., 2013). Hence, we could link these behavioral effects with the changes observed at the level of the $\mathrm{C} 1$ only indirectly. Moreover, the 
question remains whether positive mood could be associated with a gain (as opposed to a cost) regarding the processing of these peripheral stimuli at the behavioral level, when task instructions emphasize the processing of global elements or coarse information, rather than local discrimination. Presumably, if positive mood automatically broadens attention to these peripheral stimuli, then the processing of a more global property, such as location (as opposed to their local content) might be facilitated, compared to a neutral mood condition.

\section{Rationale of the present study}

To address these questions, we adapted in this study our previous paradigm (Vanlessen, et al., 2013) and we instructed participants to attend to the location of peripheral stimuli shown in the upper visual field (secondary task), besides monitoring a stream of rapidly presented stimuli in the center of the screen (primary task, RSVP identical to our previous study). Importantly, high density (i.e., 128 channels) EEG was continuously recorded enabling us to concurrently characterize changes in top-down attention control (P300 component) and early sensory processing (C1 amplitude) induced by positive mood. While central fixation was ensured by means of the primary task ${ }^{1}$, participants were asked to pay attention (peripheral vision) to the location of the peripheral stimuli shown at an unpredictable time and location relative to the central RSVP. More specifically, we asked them to detect overtly (by means of a specific button press) the appearance of textures shown randomly at a predefined target position (in the middle of the upper visual field), while ignoring the two other competing locations (either above or below the target position). This way, we could obtain a behavioral estimate of participants' ability to localize stimuli shown in the upper visual field. Given the size and shape of the peripheral textures used in our study (see Methods), this task required to

\footnotetext{
${ }^{1}$ The terms 'primary' and 'secondary' do not obey to a hierarchy among these two tasks, but these adjectives are used throughout the manuscript to make a clear distinction between the task at fixation ('primary') and the concurrent spatial localization task in the upper visual field ('secondary').
} 
process global spatial information, as opposed to their local elements, as in our previous study (Vanlessen, et al., 2013).

Following the $\mathrm{C} 1$, we also assessed whether the extrastriate $\mathrm{P} 1$ component to the peripheral stimuli might be influenced by "targetness" and positive mood. Previous studies already reported that this component, peaking around 100-150 ms after stimulus onset, was sensitive to manipulations of selective attention, being larger for attended compared to unattended stimuli (Hillyard \& Anllo-Vento, 1998; Martínez, et al., 1999). Accordingly, we could assess whether peripheral stimuli shown at the attended location in the upper visual field (i.e., the middle position) would elicit a larger P1 than those shown at the unattended locations (i.e., either below or above the middle position). We could also test whether positive mood could influence this gain control mechanism.

Moreover, for each of the three locations in the upper visual field, we also used two different texture elements to assess whether positive mood could blur the spatial resolution in the upper visual field (see Vanlessen, et al., 2013) and hence lead to a drop in their discrimination, as indexed by the N1 component (Vogel \& Luck, 2000). Although participants were not instructed to pay attention to the content of the textures (they were instructed to localize them), we reasoned that the N1 component would give us an indirect correlate of their covert discrimination in the extrastriate visual cortex. An extensive pilot testing was carried out to select two textures that were able to elicit a similar $\mathrm{C} 1$ component but a different N1 component (see Methods). If positive mood broadens attention to these peripheral stimuli while at the same time it decreases their spatial resolution, we surmised that the amplitude of the $\mathrm{N} 1$ component would be significantly less influenced by the texture content in the positive, relative to the neutral mood group.

This revised paradigm allowed us to extend our previous ERP findings (Vanlessen et al., 2013) in several ways. First, we could assess whether the early broadening of attention 
seen previously at the level of the $\mathrm{C} 1$ could be deemed "automatic" (i.e., occurring regardless of the fact that the peripheral stimuli were task-relevant or not). Possibly, making the peripheral stimuli task-relevant could impede an early modulation of the $\mathrm{C} 1$ by positive mood, because additional processes were required to treat them explicitly. Second, we could test whether the putative broadening of attention after the induction of positive mood would be accompanied by a behavioral facilitation for the processing of the spatial location of these peripheral stimuli, compared to a neutral mood condition. Third, we could explore whether stages of sensory processing subsequent to the $\mathrm{C} 1$ might also be influenced by positive mood. More specifically, we could evaluate whether selective attention to these peripheral stimuli (P1 component) and their subsequent implicit discrimination (N1 component) could also be modulated by positive mood, besides the $\mathrm{C} 1$. Finally, we could examine whether, with this dual task setting, positive mood might potentially alter the processing of the central stimuli shown during the RSVP (both at the behavioral and ERP levels).

Using this paradigm, we formulated the following predictions. (i) We hypothesized that, at the behavioral level, participants in the positive mood group would better discriminate the location of the peripheral stimuli, compared to participants in the neutral mood group. This prediction was formulated based on earlier studies linking positive affect with a preference for global information processing (Basso, Schefft, Ris, \& Dember, 1996; Gasper \& Clore, 2002; Srinivasan \& Hanif, 2010) and a broader focus of attention (Fredrickson, 2001; Moriya \& Nittono, 2011; Rowe, et al., 2007). Unlike previous studies using primarily conflict or interference tasks ( Moriya \& Nittono, 2011; Rowe, Hirsh, \& Anderson, 2007), a strength of this paradigm was the possibility to relate positive mood to a genuine benefit in the spatial localization of peripheral stimuli, consistent with a broadening of attention. (ii) Critically, we predicted that the $\mathrm{C} 1$ elicited by the peripheral stimuli would be larger in magnitude in the positive relative to the neutral mood group, indicating an early gating of attention towards 
these peripheral stimuli in the primary visual cortex that may underlie a broadening of attention in this specific mood state (Fredrickson, 2001; Vanlessen, et al., 2013). (iii) Besides the $\mathrm{C}$ 1, we also explored whether positive mood could influence selective attention to these peripheral stimuli (P1 component), as well as their implicit discrimination (N1 component). Given that the extrastriate visual P1 component varies in amplitude with selective attention (Heinze, Mangun, et al., 1994; Mangun, Buonocore, Girelli, \& Jha, 1998; Pourtois, Grandjean, Sander, \& Vuilleumier, 2004), we reckoned that this component would be larger for target textures (i.e., middle position in the upper visual field) compared to textures serving as distractors (above and below this specific location). For the subsequent N1 component, we predicted that its amplitude would vary depending on the texture content. Because we already found a drop in spatial resolution for these peripheral textures in our previous study (see Vanlessen, et al., 2013), we surmised that positive mood could blur this N1 effect. (iv) Finally, regarding the primary task, we predicted that the (oddball) target stimuli embedded in the RSVP would elicit a larger P300 compared to the standard stimuli (see Moriya \& Nittono, 2011; Rossi \& Pourtois, 2012; Vanlessen, et al., 2013 for similar findings), indicating a clear detection of these target stimuli. We also expected participants to detect most of them (see also Rossi \& Pourtois, 2012; Vanlessen, et al., 2013). Given that several previous ERP studies consistently found this P300 effect regardless of changes in the affective state of the participant, we had no reason to expect positive mood to alter this pattern for the P300 component (Rossi \& Pourtois, 2012; Vanlessen, Rossi, De Raedt, \& Pourtois, 2013). Accordingly, we did not expect changes for the primary task (both at the behavioral and ERP levels) depending on the (positive) mood. 


\section{MATERIALS AND METHODS}

\section{Participants}

Forty-two undergraduate students from Ghent University participated in this study (age: $M=$ $22 ; S D=2 ; 6$ male participants per group). According to a self-report questionnaire, all participants had normal or corrected-to-normal vision and no history of psychiatric or neurological disorders. All participants gave written informed consent prior to participation. Participants were randomly assigned to either a positive or a neutral mood condition. The data of two participants from the positive mood group were excluded from further analysis: one participant suffered from repeated migraine attacks during the experiment; another one showed excessive low accuracy for the secondary task (accuracy of $1.41 \%$ for target detection). Hence, twenty participants per mood group were included in the final sample. The study protocol was conducted in accordance with the Declaration of Helsinki and approved by the local ethics committee.

\section{Materials}

Mood Induction. Participants received either a positive or a neutral/control mood induction in a between-subjects design. The MIP used in our previous study (Vanlessen, et al., 2013) was found to be successful in eliciting the desired (positive or neutral) mood state in participants and was therefore used again in the current study. The MIP consisted of an imagery procedure in which participants were instructed to vividly imagine to re-experience an autobiographical memory (Holmes, 2006; Holmes, Coughtrey, \& Connor, 2008). The MIP was preceded by imagery exercises (i.e. holding and manipulating a lemon) in order to train participants to imagine from their own perspective (Holmes, 2006; Holmes, et al., 2008). During the MIP, participants were asked to recall and report a specific situation they experienced at least one week before the experiment, that made them feel either very happy (positive mood condition) 
or neutral (neutral mood). Next, participants closed their eyes and tried to vividly imagine reliving the reported experience for 30 seconds. Then, the experimenter asked the participants questions about the sensations they could experience during imagination, in order to encourage concrete imaginations from the requested perspective (Watkins \& Moberly, 2009; based on Holmes et al., 2008). Next, participants imagined the recalled experience for another 30 seconds. During both the memory recall and the experimental task, experimentally validated classical music fragments were playing in the background in order to implicitly trigger the associated mood (Bower \& Mayer, 1989; Mitterschiffthaler, Fu, Dalton, Andrew, \& Williams, 2007). Participants were instructed to pay no attention to the music. We ensured participants remained naïve regarding the purpose of the MIP using a cover story making them believe that the experiment concerned the relationship between the processing of visual information and the ability to use imagination.

Changes in subjective levels of mood following the MIP were measured by means of three questionnaires: the Positive and Negative Affect Schedule (PANAS; Watson, Clark, \& Tellegen, 1988), the Self-Assessment Manikin for Arousal (SAM, Bradley \& Lang, 1994) and three 10 centimeters, horizontal Visual Analogue Scales (VAS) for the feelings happiness, pleasantness and sadness. The left anchor of the VAS was labeled 'Neutral', while the right one was labeled 'As happy/pleasant/sad as you can imagine'.

Main Task. Participants performed a dual task deriving from an experimental paradigm validated previously (Rauss, Pourtois, Vuilleumier, \& Schwartz, 2009; Rossi \& Pourtois, 2012, 2013; Schwartz, et al., 2005). The primary task was used to ensure sustained central/foveal vision throughout the experimental session and measure top-down attention control mechanisms. It consisted of a RSVP of short lines $(1 \mathrm{~cm})$ at central fixation, that could be either standard lines (tilted $35^{\circ}$ counterclockwise from the vertical axis) or target lines 
(tilted $45^{\circ}$ ), with a 4:1 standard/target ratio (see Fig. 1A). Participants made a key press on a response box upon target detection (using a pre-defined key). The secondary task was decoupled from this foveal RSVP and entailed the localization of visual textures shown in the upper visual field. These stimuli were presented at an unpredictable location and time (i.e. variable SOA between central stimulus and peripheral texture) relative to the central stimuli. Central and peripheral stimuli never overlapped in space nor time.

The peripheral stimuli consisted of visual textures $\left(3^{\circ} \times 34^{\circ}\right.$ of visual angle) organized in two horizontal lines that were constituted of either crosses $(0.8 \times 0.9 \mathrm{~cm})$ or snowflake-like elements $(0.9 \times 1 \mathrm{~cm}$, see Fig. 1C), with an equal number of presentations of each type at each location (at $5.3^{\circ}$, Close; $7.8^{\circ}$, Middle; or $10.3^{\circ}$, Far from central fixation). Based on a pilot EEG study, we selected these two textures because they elicited a reliable amplitude difference at the level of the N1, indexing a rapid discrimination of the content between these two textures (i.e., the snowflake-like texture elicited a larger N1 amplitude than the crosses), while they both elicited a clear and similar C1, our main ERP component of interest. However, this variation along the texture content was task-irrelevant (and unknown to the participants), whereas the actual spatial location of the peripheral stimulus was the taskrelevant stimulus dimension (secondary task). Participants were instructed to detect overtly any peripheral texture stimulus appearing in the middle position in the upper visual field (using another pre-defined key). Thus, participants responded with one of two predefined keys of the response box for the central targets and with the other one for peripheral targets; this stimulus-response mapping was counterbalanced across participants. In addition, participants were instructed to withhold responding for textures appearing randomly at one of the two other competing positions. Thus, the non-target peripheral stimuli (i.e., textures shown either below or above this middle location, resulting in distractors appearing close to fixation or far from it; see Fig. 1B) did not require any manual response. 
Localizer. When the main task was completed, participants received two additional blocks containing peripheral stimuli only under passive viewing conditions (no RSVP at fixation; mere fixation required). These peripheral stimuli were shown at six non-overlapping positions, i.e., the same three positions/eccentricities relative to fixation as in the main task (Close, Middle and Far in the upper visual field), as well as the three symmetric positions in the lower visual field. A total of 480 stimuli ( 240 per block) were presented in random order and were equally divided over the six possible locations and with an equal number of each texture subtype at each position. Due to a technical problem, the data of one participant in the positive mood group could not be saved properly.

These two additional blocks were employed to confirm that the first Visual Evoked Potential (VEP) elicited by the peripheral textures during the main task corresponded to a reliable retinotopic C1 component (see Rossi \& Pourtois, 2012; Vanlessen, et al., 2013). Hence, we used these ERP data as an independent localizer for the $\mathrm{C} 1$ component. Following standard practice, this was achieved by contrasting upper vs. lower visual field stimulations and revealing the expected polarity reversal for this early striate component (manifested by a negative $\mathrm{C} 1$ amplitude for stimuli shown in the upper visual field, but a positive $\mathrm{C} 1$ amplitude at the same early latency following stimulus onset for the same stimuli shown in the lower visual field; see Clark, et al., 1995; Jeffreys \& Axford, 1972; Rauss, et al., 2011). These properties (latency, amplitude, polarity and topography) allowed us to confirm that the earliest component generated during the main task (peripheral stimuli) corresponded to a $\mathrm{C} 1$ component likely generated in the fundus of the calcarine fissure. A primary source of the $\mathrm{C} 1$ in V1 was further confirmed by source localization methods (see here below).

Given that the MIP was not repeated prior to these two blocks, we surmised that residual effects of positive mood ought to be minimal (see Table 1 for direct confirmation), 
and thus would no longer exert an influence on the $\mathrm{C} 1$ amplitudes (as confirmed by our ERP results, see here below). We could also have administered these localizer blocks prior to the first MIP in order to avoid any possible carryover effect of the mood. However, we did not opt for this possibility for methodological reasons, because we did not want to draw the attention of the participants to these peripheral stimuli before the start of the experimental session. We wanted to avoid a "priming" effect that might have contaminated the $\mathrm{C} 1$ recorded for the same peripheral stimuli during the main task or altered the efficiency of the cover story told to the participants during the first MIP.

Questionnaires. Participants completed three trait-related questionnaires: the Beck Depression Inventory (BDI; Beck, Steer, Ball, \& Ranieri, 1996), the BIS/BAS scales (Carver \& White, 1994) and the Dutch Resilience scale (Rs; Portzky, Wagnild, De Bacquer, \& Audenaert, 2010). These questionnaires were used to confirm balanced (low) depression level, activation/inhibition and resilience between the two groups created artificially.

\section{Procedure}

Participants were first prepared for EEG recording and then completed two practice blocks (in total containing 45 trials, of which 8 central target lines), which were repeated until $80 \%$ accuracy for central target detection was reached. Next, the positive or neutral MIP was administered, after the field perspective training phase (duration: about 20-25 minutes). The MIP was shortly repeated (for five minutes) after blocks 3 and 6, in order to maintain the targeted mood until the end of the task. At the beginning of each trial, a fixation cross was presented $(250 \mathrm{~ms})$, followed by a central stimulus $(150 \mathrm{~ms})$, which could be a standard stimulus (in 80 percent of the trials) or a target stimulus (20 percent of the trials) requiring an overt response. The central stimulus was followed by another fixation cross (displayed during 
the SOA with an average duration of $685 \mathrm{~ms}$, randomly varying between 560 to $810 \mathrm{~ms}$ ). In half of the trials, the fixation cross stayed on the screen for another $250 \mathrm{~ms}$; in the other half, a peripheral texture was briefly presented for the same period $(250 \mathrm{~ms})$ at one out of the three possible locations in the upper visual field (see Fig. 1A). Next, a fixation cross was presented for $1500 \mathrm{~ms}$ before the next trial started. Although the textures could be presented at three possible locations above fixation, participants only had to respond to textures shown in the middle position (i.e. $7.8^{\circ}$ from fixation). The task consisted of a total of 450 central stimuli (i.e., 360 standard and 90 target lines), of which 225 were followed by a peripheral stimulus (i.e., 180 after the presentation of a central standard line and 45 after a central target line). Trials were presented in a semi-random order: the first three stimuli in a block never contained a central target line, nor a peripheral texture. Instructions emphasized accuracy and speed for both the primary and secondary tasks and a reminder of the stimulus-response mapping for both the central and the peripheral stimuli was shown at the beginning of each block. Instructions also stressed central fixation to carry out the primary task, while the secondary task had to be made using peripheral vision (in the upper visual field). At the end of the main task, participants received two blocks of 240 trials of peripheral textures under passive viewing conditions (“localizer”). All stimuli were grey and presented against a uniform black background. Each participant completed 9 blocks containing 50 trials each, seated at $57 \mathrm{~cm}$ from a 19" CRT screen, with their head movements restrained by a chinrest. The task was programmed using the E-Prime Version 2 software (Psychology Software Tools, Inc., 2001).

VASs, PANAS and SAM for Arousal were administered at the beginning of the experiment (baseline measure), after each MIP and at the end of the experiment, in order to assess directional changes in positive mood after the MIP, compared to baseline. After completion of the experimental tasks, participants received questions about the stimulus 
content of the textures presented in the upper visual field: they were asked to rate how many different texture types they think were presented in the upper visual field during the main task, how certain they were about their response and to what extent they had paid attention to the texture content. Next, participants completed the three trait-related questionnaires.

\section{Analyses of behavioral data}

To verify if mood scores changed post- compared to pre-MIP, we first calculated the average values for each VAS, PANAS and SAM administered after each MIP. Next, we compared the post-MIP scores with the baseline measure for these scales by performing separate 2 (Time: baseline vs. post-MIP) x 2 (Mood: neutral vs. positive) mixed ANOVAs on the VASs, PANAS and SAM scores as well as independent samples T-tests to further establish the specificity of the mood change in the positive mood group. Mean scores for the trait-related questionnaires were compared between mood groups using independent sample T-tests.

For the oddball task at fixation (primary task), accuracy was calculated taking into account all types of errors (i.e., false alarms to standard stimuli and missed target stimuli). We used independent samples T-tests to assess differences in accuracy and mean reaction times (RTs) for correct responses between mood groups. The accuracy for the secondary task was analyzed by means of mixed ANOVAs with Position (Close, Middle or Far) as withinsubjects and Mood (Positive vs. Neutral) as between-subjects factor. Mean RTs for the detection of target textures (Middle position in the upper visual field) were analyzed using an independent samples T-test with Mood as between- subjects factor. All T-tests were twotailed.

Trials with errors for the primary task and/or RTs exceeding $\pm 2,5$ SDs above or below the individual mean RT for the primary or secondary task were excluded from further analysis. The number of removed trials was balanced between the two mood groups (positive: 
$M=3.61 \%, S D=3.08$; neutral: $M=4.84 \%, S D=4.97, t(38)=0.94, p=0.35)$. Statistical analyses were run on $95.77 \%$ of the total data. Effect sizes were reported for all analyses. More specifically, we reported partial eta squared for the ANOVAs and Cohens' d (based on the observed means and standard deviations; see Lakens, 2013) both for the independent and paired t-tests.

\section{EEG data acquisition and reduction}

EEG was continuously recorded from a Biosemi Active Two System, using $128 \mathrm{AgAgCl}$ electrodes. EEG signals were referenced online to the CMS-DRL electrodes and sampled at $512 \mathrm{~Hz}$. Vertical oculograms were recorded through additional bipolar electrodes placed respectively above and below the left eye. The data reduction method using Brain Vision Analyzer 2.0 (Brain Products GmbH, Munich, Germany) was identical for the main task and the localizer blocks.

EEG signals were referenced offline to the linked mastoids and band-pass filters between 0.016 and $70 \mathrm{~Hz}$, and a notch-filter $(50 \mathrm{~Hz})$ were applied. Next, the EEG data were segmented relative to the onset of either central or peripheral stimuli (stimulus-locked epochs; segmentation window of $160 \mathrm{~ms}$ pre- and $740 \mathrm{~ms}$ post-stimulus onset). In order to avoid contamination by the processing of and response to the target stimuli at fixation, individual ERPs for the peripheral textures were only included in the averages when they followed a central standard stimulus that did not require any response (and did not elicit a P300).

Artifacts due to eye blinks were automatically corrected by means of the standard Gratton et al. algorithm (Gratton, Coles, \& Donchin, 1983). A spherical splines procedure was used for interpolating noisy channels. The epochs were baseline-corrected using the entire 160 ms pre-stimulus interval. Epochs containing residual artifacts were semi-automatically rejected using an absolute voltage criterion of $\pm 75 \mu \mathrm{V}$ exceeding baseline. Using this 
procedure, $79 \%$ of the epochs were found to be artifact-free. For the main task, averages were calculated separately per participant for target and standard stimuli (primary task), and for peripheral stimuli at each position (Close, Middle or Far). For the localizer blocks, individual averages were calculated for the three positions above vs. below fixation.

Given its typical centro-parieto-occipital scalp distribution, the P300 component was identified at electrode positions A19, A20 and A21 (midline; with A19 corresponding to electrode Pz in the International 10-20 System and A21 to POz); A5, A18 and A17 (left hemisphere); and A32, A31 and A30 (right hemisphere). We calculated the mean amplitude of the P300 per electrode during the time window spanning from 490 to $690 \mathrm{~ms}$ post-stimulus onset for the target and standard stimuli separately (primary task). We used this specific time window because it best encompassed the P300 component for all participants over posterior parietal electrodes. For the peripheral stimuli (secondary task), a semi-automatic peak detection was applied on the individual averages in order to score the latency and amplitude of the $\mathrm{C} 1$ and $\mathrm{P} 1$ in both the main task and the localizer blocks, as well as the $\mathrm{N} 1$ in the main task (Picton, et al., 2000). Then, for each of these deflections separately, we computed the mean amplitude around the peak using a $20 \mathrm{~ms}$ interval $(10 \mathrm{~ms}$ before and $10 \mathrm{~ms}$ after the peak). The $\mathrm{C} 1$ was defined as a negative ongoing peak with the greatest amplitude between 25 and 85 ms post-stimulus onset over occipito-parietal electrode positions A19/Pz, A20 and A21/ POz (midline); A5, A17 and A18 (left hemisphere); and A32, A31 and A30 (right hemisphere). The P1 component was defined as the first positive deflection following the $\mathrm{C} 1$, peaking between 75 and $150 \mathrm{~ms}$ post-stimulus onset. The P1 was measured at slightly more occipital (lower) electrode positions compared to the C1: A21/ POz, A22 and A23/Oz (midline); A15/O1, A16, A17 and (left hemisphere); and A28/O2, A29, A30 (right hemisphere). Finally, the N1 component was identified as the first negative wave following the P1, reaching its highest amplitude between 155 and $215 \mathrm{~ms}$ post-stimulus onset at 
electrode positions A8, A9, A10/PO7; B11/P8, B12 and B13 on the left hemisphere; and B5, B6, B7/PO8, D29, D30 and D31/P7 on the right hemisphere. These electrode positions were selected based on the topographical properties of the current data set. Statistical analyses were performed on the amplitude values pooled across the selected electrodes.

Separate mixed ANOVAs were used for the analysis of the mean amplitudes of the P300, C1, P1 and N1 components. For the P300, we used a mixed ANOVA with Stimulus (Standard vs. Target) as within-subjects factor, and Mood (Positive vs. Neutral) as betweensubjects factor. The $\mathrm{C} 1$ (main task and localizer) and P1 data were submitted to mixed ANOVAs with Position (Close, Middle or Far) as within-subjects factor, and Mood (Positive vs. Neutral) as between-subjects factor. For the analysis of the N1, we applied a mixed ANOVA with Position (Close, Middle or Far) and Texture (Crosses vs. Snowflakes) as within-subjects factors, and Mood (Positive vs. Neutral) as between-subjects factor. Twotailed paired or independent samples T-tests were used to perform post-hoc comparisons. We also performed ANOVAs on the peak latencies of these ERP components. However, these analyses did not show significant effects of mood on their latencies (all $p>.18$ for main or interaction effects involving mood as a factor). Therefore, we report the results obtained for the mean amplitudes of these components only. Whenever normality assumptions were violated, corrected p-values were used. As was the case for the analyses of the behavioral data, partial eta squared and Cohens' d were reported.

Finally, to corroborate the assumption of generators located primarily in the striate visual cortex for the $\mathrm{C} 1$ component, we used standardized low-resolution brain electromagnetic tomography (sLORETA, Pascual-Marqui, 2002). sLORETA solutions are computed within a three-shell spherical head model co-registered to the MNI152 template (Mazziotta, et al., 2001). sLORETA estimates the 3-dimensional intracerebral current density distribution in 6239 voxels ( $5 \mathrm{~mm}$ resolution), each voxel containing an equivalent current 
dipole. This 3-dimensional solution space in which the inverse problem is solved is restricted to the cortical gray matter. The head model for the inverse solution uses the electric potential lead field computed with a boundary element method applied to the MNI152 template (Fuchs, Kastner, Wagner, Hawes, \& Ebersole, 2002). Scalp electrode coordinates on the MNI brain are derived from the international 5\% system (Jurcak, Tsuzuki, \& Dan, 2007). A direct statistical comparison between the two groups for the $\mathrm{C} 1$ component was carried out using a stringent non-parametric randomization test (relying on 5000 iterations).

\section{RESULTS}

Changes in mood: manipulation check

The 2 (Time) x 2 (Mood) ANOVA on the VAS scores showed a significant interaction effect between Time and Mood for feelings of happiness (baseline: positive: $M=5.42, S D=2.72$, neutral: $M=4.51, S D=2.84$; post-MIP: positive: $M=7.87, S D=1.71$, neutral: $M=4.17$, SD $=2.74 ; F(1,38)=58.83, p<.001, \eta p^{2}=0.61$ ), and pleasantness (baseline: positive: $\mathrm{M}=5.76$, $S D=2.60$, neutral: $M=4.76, S D=2.76$; post-MIP: positive: $M=7.62, S D=1.76$, neutral: $M$ $=4.41, S D=2.50 ; F(1,38)=17.84, p<.001, \eta p^{2}=0.32$ ), but not for sadness (baseline: $M=$ $0.50, S D=0.80$, post-MIP: $M=0.77, S D=0.91, F(1,38)=1.48, p=.23, \eta p^{2}=0.04$; see Table 1 for a break-down of the happiness vs. sadness mean values obtained for the different measurement moments and groups, separately). Next, we compared VAS scores between the positive and the neutral mood groups at baseline vs. post-MIP, using independent T-tests. As expected, post-MIP mood measurements showed a significant difference between the positive and the neutral mood group for both feelings of happiness $(t(38)=5.13, p<.001, d=1.62)$ (see Fig. 2A) and pleasantness $(t(38)=4.69, p<.001, d=1.48)$ (see Fig. 2B). Importantly, at baseline, groups did not differ for reported happiness $(t(38)=1.04, p=.25, d=0.33)$ nor 
pleasantness $(t(38)=1.17, p=.95, d=0.37)$. These results show a selective increase in positive affect after MIP in the positive, but not in the neutral, mood group (see also Table 1).

The 2 (Time) x 2 (Mood) ANOVA on the PANAS scores showed a significant interaction effect between Time and Mood for the PA scales (baseline: positive: $M=31.60$, $S D=5.58$, neutral: $M=30.95, S D=6,72$; post-MIP: positive: $M=32.95, S D=6.02$, neutral: $\left.M=28.77, S D=6.98, F(1,38)=7.00, p=.012, \eta p^{2}=0.16\right)$, but not for the NA scales (baseline: $M=12.25, S D=2.33$; post-MIP: $M=11.43, S D=1.89, F(1,38)=2.56, p=.118$, $\left.\eta p^{2}=0.06\right)$. An independent samples T-test showed a significant difference between the positive and neutral mood group on the post-MIP PA scores, $\mathrm{t}(38)=2.03, p=.05, d=0.64$ (see Fig. 2C). At baseline, this difference was not significant $(\mathrm{t}(38)=0.33, p=.65, d=0.11)$. These results show that while the positive affect in the neutral mood group substantially decreased from baseline to post-MIP, no similar blunting of positive affect was seen in the positive mood group.

The 2 (Time) x 2 (Mood) ANOVA on the SAM for Arousal scores showed a significant interaction effect between Time and Mood (baseline: positive: $M=4.22, S D=$ 1.22, neutral: $M=3.63, S D=1.61$; post-MIP: positive: $M=5.22, S D=1.75$, neutral: $M=$ $\left.3.21, S D=1.32, F(1,35)=8.52, p=.006, \eta p^{2}=0.20\right)$. An independent samples T-test on the Arousal scores showed a significant difference between the positive and neutral mood group (with higher subjective levels of arousal in the former compared to the latter group), $t(35)=$ 4.20, $p=.001, d=1.30$ after the MIP, but not at baseline, $t(35)=1.26, p=.22, d=0.41$.

\section{Behavioral results.}

Primary task

Participants reached high levels of accuracy, equally so in each mood group (positive group: $M=96.65, S D=2.93$; neutral group: $M=96.51, S D=3.62, t(38)=0.13, p=.90, d=0.04)$. 
Likewise, RTs for correct target detection did not differ significantly between groups, although the RTs in the positive group were numerically shorter (positive group: $M=$ 482.866, $S D=85.39$; neutral group: $M=524.21, S D=69.27, t(38)=1.68, p=.10, d=0.53$ ). Together, these results show a balanced performance between the two mood groups for the primary task, confirming one of our predictions. Moreover, the high accuracy in each group suggests that participants maintained fixation in the center of the screen throughout the experimental session and processed the textures in the upper visual field with peripheral vision (see also Rossi \& Pourtois, 2013).

\section{Secondary task}

A mixed ANOVA performed on the mean accuracy scores with Position and Group as factors showed a significant effect of Position (Close: $M=91.33, S D=8.64$; Middle: $M=84.16, S D$ $=14.64$, Far: $\left.M=86.24, S D=13.26, F(2,76)=4.02, p=.022, \eta \mathrm{p}^{2}=0.10\right)$. The interaction effect of Position and Group $\left(F(2,76)=0.93, p=.40, \eta \mathrm{p}^{2}=0.02\right)$, as well as the main effect of Group $\left(F(1,38)=0, p=.99, \eta \mathrm{p}^{2}<.001\right)$, were not significant. A post-hoc independent samples T-test performed on the RTs for target detection showed that participants in the positive mood group $(M=593.79, S D=108.14)$ identified the target textures faster than participants in the neutral mood group $(M=667.72, S D=82.76, t(38)=2.43, p=.020, d=0.77)$. These results confirmed our second prediction.

To evaluate the presence of a possible trade-off between the primary and the secondary task, we performed an auxiliary correlation analysis showing however no relation between them regarding accuracy $(r=.082, p=.61)$. A positive relation was found $(r=.36, p$ $=.023$ ) for the speed with these two tasks, indicating that participants who were faster with the primary task were also faster with the secondary. Hence, these results confirm that high 
accuracy for the primary task was not compensated by low accuracy for the secondary task or vice versa.

Primary and secondary task combined

We also performed an ANOVA on these RT data with Mood as between-groups factor and Task as within-groups factor. The rationale for this control analysis was to assess whether the substantial RT facilitation $(d=0.77)$ as a function of positive mood observed for the secondary task could be dissociated from the (non-significant) RT facilitation found for the primary task with positive mood. This analysis showed significant main effects of Task $\left(F(1,38)=72.04, p<.001, \eta p^{2}=0.66\right)$ and $\operatorname{Group}\left(F(1,38)=6.31, p=.0,6 \eta p^{2}=0.14\right) ;$ however, the interaction effect between the two factors failed to reach significance $(F(1,38)=$ $\left.1.30, p=.26, \eta p^{2}=0.03\right)$. Moreover, using $G^{*}$ Power, we estimated that we would need to test at least 64 participants per group to achieve a significant group difference for the primary task, with a medium effect size $(d=0.50)$ and a power of 0.80 . Accordingly, these results suggest that positive mood likely led to a general RT facilitation (including the primary and secondary tasks), however, we lacked sufficient power to reveal this effect for the primary task.

Questionnaires

No significant group differences were found regarding the trait-related variables, including the BIS/BAS (BIS: $t(38)=0.36, p=.72, d=0.11$, BAS Drive $t(38)=0.58, p=.56, d=0.18$, BAS Fun: $t(38)=0.10, p=.92, d=0.03$, BAS Reward: $t(38)=0.43, p=.67, d=0.14)$ and the Dutch Resilience Scale $(t(38)=0.35, p=.73, d=0.11)$. These results suggest that group differences found at the behavioral and ERP levels were not confounded by obvious personality differences between the two groups. However, a post-hoc independent samples T- 
test performed on the BDI scores did show a significant group difference, with a somewhat surprising higher level of (sub clinical) depression in the positive mood group $(M=9.85, S D$ $=8.02)$, compared to the neutral mood group $(M=4.80, S D=4.54 ; t(38)=2.45, p=.019, d=$ 0.77). However, this unexpected group difference regarding levels of depression did not prevent participants in the positive mood group to increase their positive mood following the MIP, compared to participants assigned to the neutral mood group (see behavioral results here above). Furthermore, these BDI scores did not correlate with behavioral performance (RT and ACC) for the primary or secondary task, nor with the P300 to central targets or $\mathrm{C} 1$ to peripheral stimuli (all $\mathrm{p}>.24)$.

Post-experiment questions related to the texture content showed balanced results between the two groups. In each mood group, eight out of 20 participants correctly estimated the number of different textures $(n=2)$ that appeared in the upper visual field during the main task. The level of confidence in their estimation was also balanced between the two groups $(M$ $=4.04, S D=2.39 ; t(38)=1.51, p=.14, d=0.48)$, as was their subjective rating regarding the extent to which they paid attention to the texture content $(M=3.08, S D=2.16 ; t(38)=1.25, p$ $=.22, d=0.40)$.

ERP results.

Primary task

P300. The mixed ANOVA showed, as expected, a significant main effect of Stimulus type $\left(F(1,38)=211.78, p<.001, \eta \mathrm{p}^{2}=0.85\right)$. A post-hoc paired samples T-test showed that the P300 amplitude elicited by target stimuli $(M=4.27, S D=0.67)$ was significantly larger than the P300 to standard stimuli $(M=1.75, S D=0.28, t(38)=12.83, p<.001, d=2.44)$, indicating that oddball target stimuli were detected and processed differently compared to standards. 
Additionally, the ANOVA also showed a significant interaction effect between Stimulus type and $\operatorname{Mood}\left(F(1,38)=12.19, p=.001, \eta \mathrm{p}^{2}=0.24\right)$. This interaction was driven by a larger P300 for the target stimuli in the positive mood group $(M=13.34, S D=3.94)$ compared to the neutral mood group $(M=9.55, S D=3.78, t(38)=3.11, p=.004, d=0.98)$, while the P300 amplitude to the standard stimuli did not differ between groups $(t(38)=0.043$, $p=.97, d=0.01$, see Fig. 3). This interaction suggests that participants in the positive mood group likely detected targets more easily or better than the neutral mood group. However, as reported here above, this neurophysiological effect was not accompanied by a change at the behavioral level (balanced accuracy and mean RTs for the primary task between the two groups).

\section{Secondary task}

C1. The mixed ANOVA revealed a significant main effect of Position $(F(2,76)=16.95, p<$ $\left..001, \eta \mathrm{p}^{2}=0.31\right)($ Fig. 4A and B). Planned comparisons showed a drop in C1 amplitude with increasing eccentricity (see also Vanlessen, et al., 2013). The C1 was larger in magnitude for peripheral stimuli shown at the Close position $(M=-4.03, S D=2.32)$ relative to both the Middle position $(M=-1.95, S D=1.91 ; t(39)=4.94, p<.001, d=0.98)$, and the Far position $(M=-2.12, S D=1.95 ; t(39)=4.74, p<.001, d=0.89)$. The $\mathrm{C} 1$ did not differ between the Middle and Far position $(t(39)=0.49, p=.63, d=0.09)$. Importantly, this analysis also showed a significant main effect of Mood, showing a greater $\mathrm{C} 1$ amplitude to peripheral stimuli (regardless of their position) in the positive $(M=-3.24, S D=1.46)$ compared to the neutral mood group $\left(M=-2.16, S D=1.35, F(1,38)=5.95, p=.019, \eta \mathrm{p}^{2}=0.14\right)($ see Fig. 4$)$.

The independent localizer blocks enabled us to ascertain that this early component recorded during the main task was a $\mathrm{C} 1$ component. A direct (visual) comparison between the main task and localizer blocks showed that the $\mathrm{C} 1$ had the same morphology and topography 
in these two sessions. Second, we found a clear-cut polarity reversal for this component depending on which part of the visual field (either upper or lower) was stimulated with these textures (see Fig. 5A and B), confirming that this early component had a striate origin (Kelly, et al., 2013). The ANOVA performed on the mean C1 amplitudes for the stimuli presented in the upper visual field during the localizer blocks yielded a significant main effect of position $\left(F(2,74)=19.94, p<.001, \eta \mathrm{p}^{2}=0.35\right)$, suggesting a linear decrease of the $\mathrm{C} 1$ amplitude with increasing eccentricity (Close vs. Middle: $t(38)=4.61, p<.001, d=0.85$; Close vs. Far: $t(38)$ $=5.58, \mathrm{p}<.001, d=1.23$; Middle vs. Far: $t(38)=2.21, p=.03, d=0.48)$. Unlike the main task, no main effect of $\operatorname{Mood}\left(F(2,74)=0, p=.99, \eta \mathrm{p}^{2}<0.001\right)$, nor an interaction effect between Position and $\operatorname{Mood}\left(F(2,74)=1.59, p=.21, \eta \mathrm{p}^{2}=0.04\right)$ was found for the $\mathrm{C} 1$ in this analysis (localizer blocks), suggesting that when the MIP was ceased, modulatory effects of positive mood on this early retinotopic component were no longer present.

For the main task, results of the source localization algorithm (sLoreta) confirmed that the larger $\mathrm{C} 1$ in the positive compared to the neutral mood group was associated with enhanced activity in the primary visual cortex (see Fig. 4D).

P1. The mixed ANOVA showed a main effect of position $\left(F(2,76)=6.57, p=.002, \eta \mathrm{p}^{2}=\right.$ $0.15)$, in such a way that the Close $(M=6.32, S D=3.07)$ and Middle position $(M=6.08, S D$ $=3.13)$ led to a larger P1 than the Far position $(M=4.91, S D=2.75 ; t(39)=2.98, p=.005, d$ $=0.48$; and $t(39)=3.12, p=.003, d=0.40$ for these two comparisons, respectively) (see Fig. 5C). The amplitude of the P1 was balanced for the Close and Middle positions $(t(39)=0.59, p$ $=.56, d=0.08)$.

The observation that the amplitude of the P1 did not decrease monotonically with increasing eccentricity (like the $\mathrm{C} 1 \mathrm{did}$ ) is compatible with earlier studies that have reported enhanced P1 effect for attended vs. unattended stimuli (Anllo-Vento, Luck, \& Hillyard, 1998; 
Martínez, et al., 1999). Attended stimuli were textures shown in the middle position of the upper visual field in the present case. For this location, the P1 was reliably larger than for the Far position (where no overt discrimination was required). However, the P1 was equally large in size for the Middle and Close positions. This could be explained by the fact that in our experimental design, attention had to be allocated to two non-overlapping positions concurrently (i.e., the center of the screen and the middle part of the upper visual field). In these conditions, visual stimuli appearing between these two anchors (textures shown at the Close position) somehow received priority alike during the competition for attention selection (Castiello \& Umilta, 1992; Heinze, Luck, et al., 1994; Jans, Peters, \& De Weerd, 2010). At any rate, the results obtained for the P1 clearly showed that the Middle position was somehow prioritized or attended relative to the Far position, given the specific task demands (secondary task).

Unlike the $\mathrm{C} 1$ component, no significant main effect of Mood was found for the P1 component $\left(F(1,38)=0.24, p=.63, \eta \mathrm{p}^{2}=0.006\right)$, nor a significant interaction effect between Mood and Position $\left(F(2,76)=1.41, p=.25, \eta p^{2}=0.04\right)($ see Fig. 4 and 5C)

N1. The mixed ANOVA yielded a significant main effect of Texture $(F(1,38)=16.17, p<$ $.001, \eta \mathrm{p}^{2}=0.30$ ) (see Fig. 5D). However, no main effect of Position or Mood, nor interaction effects between these factors reached significance (all $p>.05$; all $\eta \mathrm{p}^{2} \leq 0.05$ ). This finding suggests that although the texture content was kept task-irrelevant, the two textures elicited a differential activation (presumably in the extrastriate visual cortex) early on following stimulus onset, at the level of the N1 (Luck, Woodman, \& Vogel, 2000; Vogel \& Luck, 2000). However, positive mood did not alter this early discrimination process (as we surmised it might have been the case if a broadening of attention under positive mood would also be accompanied by a drop in spatial resolution). 


\section{DISCUSSION}

In this study, we sought to investigate, using ERP measurements, whether positive mood could influence the early retinotopic processing (indexed by the $\mathrm{C} 1$ component; see Rauss, et al., 2011) of attended and task-relevant stimuli shown in the upper visual field at various locations/eccentricities relative to fixation, in agreement with a broadening of spatial attention associated with this specific mood state (Ashby, Isen, \& Turken, 1999; Fredrickson, 2001, 2004). To test this prediction, participants were assigned either to a neutral/control or positive mood condition, and they performed a dual task involving an oddball detection at fixation and a localization task in the periphery of the upper visual field, while high-density EEG was recorded concurrently. Results showed that our MIP was efficient and successful to elicit a reliable and sustained change in positive affect (see Table 1). Positive mood influenced target processing at fixation (primary task), indicated by a larger P300 component. Importantly, the $\mathrm{C} 1$ component to all peripheral textures (secondary task) was substantially enhanced in the positive compared to the neutral mood group, regardless of whether the textures were targets or not. Moreover, in the positive mood group, participants detected targets faster than in the neutral mood group. No differential effect of positive mood was found for the subsequent P1 or N1 component. However, these two extrastriate components varied in amplitude in a predictive way: while the P1 was enhanced for attended relative to unattended stimuli (Heinze, Mangun, et al., 1994; Hillyard \& Anllo-Vento, 1998), the amplitude of the N1 changed depending on the texture content (Vogel \& Luck, 2000). These results suggest that positive mood can boost the early spatial encoding of the peripheral stimuli in the primary visual cortex selectively, before top-down attention control mechanisms gate sensory processing in the extrastriate visual cortex. Hereafter, we discuss the implications of these new neurophysiological results. 


\section{Early and automatic broadening of attention with positive mood}

Our results show that positive mood influenced the earliest cortical stage of stimulus processing in $\mathrm{V} 1$, indexed by the $\mathrm{C} 1$. As expected, the amplitude of the $\mathrm{C} 1$ reliably decreased with increasing eccentricity relative to fixation (Di Russo, Martínez, Sereno, Pitzalis, \& Hillyard, 2002; Jeffreys \& Axford, 1972; Rauss, et al., 2011). This result indirectly suggests that foveal vision was used to process the RSVP at fixation (primary task), and that the peripheral stimuli shown in the upper visual field (secondary task) were therefore processed with peripheral vision. Additional source localization analyses confirmed that the $\mathrm{C} 1$ component had a striate origin. Strikingly, the C1 was substantially larger in the positive compared to the neutral mood group uniformly across the three positions used in the upper visual field, despite the fact that only one of them (i.e., the Middle one) had actually to be attended. At the behavioral level and consistent with one of our predictions, we found that participants in the positive mood group discriminated the location of these peripheral stimuli faster than in the neutral mood group, although without a gain in accuracy. Accordingly, the putative broadening of attention following the induction of positive mood seems to be associated with a facilitation in processing the location of peripheral stimuli. However, the fact that this RT facilitation (secondary task) could not be dissociated from a general speeding up during target processing (see behavioral results) suggests that positive mood had probably larger effects than we hypothesized, and it also influenced target processing at fixation (primary task).

The new findings obtained for the $\mathrm{C} 1$ component suggest in turn an early and automatic boost of the early spatial encoding of these textures in the positive compared to the neutral mood group, which might eventually underlie a broadening of spatial attention in this specific mood state (Fredrickson, 2001, 2004). They also extend our previous results showing 
that a broadening of spatial attention captured by this early $\mathrm{C} 1$ component can also be observed when the peripheral stimuli are directly task-relevant, and not simply used as distractors (Vanlessen, et al., 2013; see also Table 2). Importantly, this early mood-dependent $\mathrm{C} 1$ effect was evidenced before top-down attention control mechanisms operating at the level of the P1 and yielding classical gain control effects for attended, relative to unattended stimuli took place (Hillyard \& Anllo-Vento, 1998; Martínez, et al., 1999). Unlike the preceding C1, the amplitude of the P1 was not modulated by positive mood, but rather by the targetness of the peripheral stimuli (and hence the amount of selective attention presumably allocated to them). In accordance with our prediction, our results showed that the P1 was enhanced for the Middle position, compared to the Far position, in line with research showing increased P1 amplitudes for attended compared to unattended stimuli in conditions of sustained attention (Heinze \& Mangun, 1995). Interestingly, unattended stimuli at the Close position also elicited an enhanced P1 component. This result can be explained by the fact that peripheral textures shown at this location appeared between the main focus of attention in the center of the screen (primary task) and the secondary focus of attention likely anchored in the middle of the upper visual field (secondary task). Because spatial attention cannot be split into two independent or separate foci simultaneously (Castiello \& Umilta, 1992; Heinze, Luck, et al., 1994; Jans, et al., 2010), these stimuli, like the ones shown at the (attended) Middle position, received enhanced processing in the extrastriate visual cortex (P1 effect), compared to the ones shown at the Far position.

Altogether, these ERP results suggest a dissociation between an early automatic broadening of attention taking place in the striate cortex (C1) under positive mood, and topdown attention gain control mechanisms operating in the extrastriate visual cortex (P1) at a later latency following stimulus onset and independent of mood. Even though the results obtained for the $\mathrm{C} 1$ (and P1 to a lesser degree) suggest that these peripheral stimuli were 
processed with peripheral vision, we did not monitor however the actual position of the eyes during the experiment, and therefore we cannot formally exclude the possibility of saccadic eye movements towards the upper visual field. To remedy this limitation, future studies should include the online monitoring of the eye position (using eye-tracking methods).

As predicted, the subsequent N1 component was influenced by the type of textures shown in the upper visual field (with two types shown equally often in random order), however, this effect was not influenced by positive mood, as we had predicted. These results suggest that a rapid and implicit discrimination occurred between these two texture types (Vogel \& Luck, 2000), equally so in each group. Contrary to one of our predictions, we did not find evidence for a reduced spatial resolution (and hence diminished N1 discrimination process) accompanying the broadening of attention after the induction of positive mood. In our previous study (Vanlessen, et al., 2013), such an effect was found when the content (rather than the position) of the peripheral stimuli became task-relevant. Hence, our results suggest that the likely tradeoff effect between a broadening of attention and a reduced spatial resolution (Castiello \& Umilta, 1990; Eriksen \& Yeh, 1985; Ivry \& Robertson, 1998) following the induction of positive mood could entail different processing stages following stimulus onset. Whereas the broadening of attention would mainly involve the early and automatic retinotopic encoding of the stimuli in the striate cortex ( $\mathrm{C} 1$ component), the concurrent diminished spatial resolution would concern later processing stages indexed by the $\mathrm{P} 1$ and N1 components, which are known to be generated after the $\mathrm{C} 1$ component in the extrastriate visual cortex (Martínez, et al., 1999).

The observation of a component specific modulation of early sensory processing by positive mood (here at the level of the $\mathrm{C} 1$ ) might be explained by the use of a simple task (secondary task), mostly tapping into spatial localization abilities in the present case. Because the amplitude of the $\mathrm{C} 1$, unlike the subsequent $\mathrm{P} 1$ or $\mathrm{N} 1$, is primarily sensible to the position 
of the stimulus in the visual field (as opposed to its content), it is therefore not entirely surprising to find a modulation of this early component, selectively. Should we have used other task demands (focusing for example on the content rather than the position of these peripheral stimuli), maybe the experimental outcome would have been then slightly different, with modulations of the P1 and N1 amplitude by positive mood alike, besides the $\mathrm{C} 1$. A modulation of the P1 component following the induction of positive mood was already reported previously (Moriya \& Nittono, 2011). On the other hand, given that we already found a similar selective modulation of the $\mathrm{C} 1$ component by positive mood when no task was required with these peripheral stimuli (see Vanlessen, et al., 2013; see also Table 2), we are inclined to conclude that these amplitude modulations of early sensory processing in V1 (C1 effect) are deemed "automatic", in the sense of occurring prior to and independently from later attention gain control or task effects (usually occurring at the level of the P1 or N1 in the extrastriate visual cortex). In this framework, positive mood would therefore be associated with a short, "phasic" broadening of spatial attention, the locus of which would be restricted to the first sweep of activation in the primary visual cortex following stimulus onset. Whether or not this early gating effect in V1 by positive mood is compatible with the concurrent modulation of specific long-distance neural pathways (connecting the prefrontal cortex and amygdala to the occipital lobe; see Pourtois, Schettino, \& Vuilleumier, 2013) or neurotransmitter systems (e.g., dopaminergic-related; see Ashby, Isen, \& Turken, 1999) remains an open question for future research.

More generally, our new results add to the growing literature showing systematic amplitude variation of this early retinoptic component not only as a function of changes in (top-down) attention control mechanisms (Kelly, Gomez-Ramirez, \& Foxe, 2008; Rauss, et al., 2009), but also the current mood state of the participant or specific emotional factors (Pourtois, et al., 2004; Rossi \& Pourtois, 2012; Stolarova, Keil, \& Moratti, 2006; Weymar, 
Keil, \& Hamm, 2013). These amplitude changes during early sensory processing in the primary visual cortex by emotion or mood occurring at the level of the $\mathrm{C} 1$ all share in common the fact that they are fast, usually component specific and orthogonal to more classical effects of load or selective attention (Handy, Soltani, \& Mangun, 2001). As such, they could be deemed "automatic" to some extent (Moors \& De Houwer, 2006), and likely occur via dynamic modulations or plasticity in specific neural routes connecting mesiotemporal lobe structures and the ventral prefrontal cortex to the occipital lobe, including the primary visual cortex (Amaral, Behniea, \& Kelly, 2003; Gschwind, Pourtois, Schwartz, Van De Ville, \& Vuilleumier, 2012; Pourtois, Schettino, \& Vuilleumier, 2013; Vuilleumier, 2005).

\section{Positive mood influences top-down attention and early sensory processes independently}

Besides the $\mathrm{C} 1$ to the peripheral stimuli, we also found that positive mood influenced target processing at fixation (primary task), indicated by a larger P300 for participants in the positive than the neutral mood group. This result was unexpected. Because in each group target stimuli elicited a much larger P300 than standard stimuli (see Fig. 3), this results suggests an enhanced processing of these target stimuli in the positive group (Kim, Kim, Yoon, \& Jung, 2008; Kok, 2001; McCarthy \& Donchin, 1981; Sawaki \& Katayama, 2007), even though this neurophysiological effect did not translate into a gain in accuracy or RT speed. This finding suggests that positive mood was associated either with an enhanced efficiency or fluency (or less effortful control) during the primary task (see Polich, 2007), or alternatively, that because arousal (besides positive emotion) was also augmented in the positive mood group, the target P300 was in turn increased in amplitude in this group (Nieuwenhuis, Aston-Jones, \& Cohen, 2005; Polich \& Kok, 1995). In our study, we did not find, however, a positive relation between changes in subjective levels of arousal following the MIP and the amplitude of the P300 to the target stimuli (primary task). Nonetheless, future studies are needed to assess the 
specific or respective contribution of arousal vs. positive valence on the observed neurophysiological effects (C1 and P300).

It is important to mention that effects of positive mood on the early sensory processing of the peripheral stimuli ( $\mathrm{C} 1$ component) cannot be explained by a general modulatory effect of this mood state that would influence the $\mathrm{C} 1$ to these peripheral stimuli and the P300 to the central targets equally or uniformly. First, at the behavioral level, we did not find evidence for a tradeoff between the two tasks. Moreover, we included and analyzed VEPs (C1, P1 and N1) to the peripheral stimuli if and only if they followed (central) standard stimuli (that did not require any response and were associated with a reduced P300 component of similar size in both groups). Accordingly, our results ( $\mathrm{C} 1$ component) are not confounded by potential lingering ERP activities or carry-over effects from the preceding target-related (central) stimulus (P300 effect). In this context, influences of positive mood on the early sensory processing of the peripheral stimuli ( $\mathrm{C} 1$ component, corresponding to a broadening of attention) are orthogonal to changes in target processing with this specific mood state (P300 component). To lend further support to this claim, we submitted the amplitude values of the $\mathrm{C} 1$ and P300 component to the same ANOVA, including three factors: Task (primary vs. secondary), Condition (non-target vs. target), and Group (positive vs. neutral) ${ }^{2}$. We reasoned that if the effects of positive mood are dissociable for the central (primary task) and peripheral stimuli (secondary task), then this control analysis should reveal a significant interaction effect between these factors. The results showed a significant three-way interaction $(F(1,38)=$ $5.75, p=.02, \eta \mathrm{p}^{2}=0.13$ ), confirming that (positive) mood did not boost ERP activity in general, but dissociable effects were evidenced for the primary and secondary task. Whereas positive mood enhanced the $\mathrm{C} 1$ component to all peripheral stimuli regardless of their "targetness" (indicated by a significant main effect of Group; see ERP results for the

\footnotetext{
${ }^{2}$ We used the amplitude of the P300 for the primary task, whereas the absolute amplitude values of the $\mathrm{C} 1$ were used for the secondary task. The non-target condition refers to the standard stimuli in the primary task, and the positions Close and Far in the secondary task.
} 
secondary task), it did enhance the P300 for the central stimuli, but only when they were targets (indicated by a significant interaction effect between Group and Condition; see ERP

results for the primary task ). Combined together, these results suggest that positive mood can probably exert effects on stimulus processing and attention control mechanisms at multiple levels and through specific modulations in distinct neural networks.

\section{CONCLUSIONS}

Our new ERP results show that positive mood can lead to a boost in early sensory processing in V1 (C1 component) related to the spatial position of task-related peripheral stimuli, selectively. A larger $\mathrm{C} 1$ component in the positive than neutral mood group is consistent with the broaden-and-build-theory (Fredrickson, 2001, 2004). This gain in spatial perception and in turn broadening of attention following the induction of positive mood can be seen as automatic because it is rapid, not modulated by task demands, and it takes place before topdown attention gain control mechanisms come into play and eventually gate the processing of attended compared to unattended locations or stimuli (P1 effect). Moreover, positive mood was found to influence the processing of central target stimuli (P300), irrespective of these changes in early sensory processing for the peripheral stimuli (C1). Altogether, these findings bolster the assumption that positive mood may broaden spatial attention by means of modulatory effects of sensory processing in V1 rapidly following stimulus onset. Whether these modulatory effects depend on specific neurotransmitter systems (e.g., dopamine; see Ashby, et al., 1999) or not remains an unanswered question. Moreover, additional imaging studies are needed to better characterize the neural pathways likely involved in these early mood-dependent sensory modulations in the primary visual cortex, given that their sources or origins might very well implicate remote and distant brain regions in the limbic system and prefrontal cortex (Pessoa, 2008; Pourtois, et al., 2013; Vuilleumier, 2005). 


\section{ACKNOWLEDGEMENTS}

This worked is supported by a Concerted Research Action Grant from Ghent University awarded to RDR and GP (\#BOF10/GOA/014). GP is funded by the European Research Council (Starting Grant \#200758) and Ghent University (BOF Grant \#05Z01708). VR is funded by a BOF grant from Ghent University (\#BOF13/PDO/095). The authors have no conflict of interest to declare.

\section{REFERENCES}

Amaral, D. G., Behniea, H., \& Kelly, J. L. (2003) Topographic organization of projections from the amygdala to the visual cortex in the macaque monkey. Neuroscience, 118, 1099-1120.

Anllo-Vento, L., Luck, S. J., \& Hillyard, S. A. (1998) Spatio-temporal dynamics of attention to color: Evidence from human electrophysiology. Hum Brain Mapp, 6, 216-238.

Ashby, F. G., Isen, A. M., \& Turken, U. (1999) A neuropsychological theory of positive affect and its influence on cognition. Psychol Rev, 106, 529-550.

Basso, M. R., Schefft, B. K., Ris, M. D., \& Dember, W. N. (1996) Mood and global-local visual processing. J Int Neuropsychol Soc, 2, 249-255.

Beck, A. T., Steer, R. A., Ball, R., \& Ranieri, W. (1996) Comparison of Beck Depression Inventories -IA and -II in psychiatric outpatients. J Pers Assess, 67, 588-597.

Botvinick, M., Braver, T. S., Barch, D. M., Carter, C. S., \& Cohen, J. D. (2001) Conflict monitoring and cognitive control. Psychol Rev, 108, 624-652.

Bower, G. H., \& Mayer, J. D. (1989) In Search of Mood-Dependent Retrieval. Journal of Social Behavior and Personality, 4, 121-156. 
Bradley, M. M., \& Lang, P. J. (1994) Measuring emotion: the Self-Assessment Manikin and the Semantic Differential. J Behav Ther Exp Psychiatry, 25, 49-59.

Bruyneel, L., van Steenbergen, H., Hommel, B., Band, G. P., De Raedt, R., \& Koster, E. H. (2013) Happy but still focused: failures to find evidence for a mood-induced widening of visual attention. Psychol Res, 77, 320-332.

Carver, C. S., \& White, T. L. (1994) Behavioral-Inhibition, Behavioral Activation, and Affective Responses to Impending Reward and Punishment - the Bis Bas Scales. $J$ Pers Soc Psychol 67, 319-333.

Castiello, U., \& Umilta, C. (1990) Size of the attentional focus and efficiency of processing. Acta Psychol, 73, 195-209.

Castiello, U., \& Umilta, C. (1992) Splitting Focal Attention. J Exp Psychol, 18, 837-848.

Clark, V. P., Fan, S., \& Hillyard, S. A. (1995) Identification of early visual evoked potential generators by retinotopic and topographic analyses. Hum Brain Mapp, 2, 170-187.

Corbetta, M., \& Shulman, G. L. (2002) Control of goal-directed and stimulus-driven attention in the brain. Nature Rev Neurosci, 3, 201-215.

Derryberry, D., \& Tucker, D. M. (1994) Motivating the focus of attention. In: Neidenthal, P. M. \& Kitayama, S. (Eds.) The heart's eye: emotional influences in perception and attention. (pp. 167-196). San Diego, CA: Academic.

Di Russo, F., Martínez, A., Sereno, M. I., Pitzalis, S., \& Hillyard, S. A. (2002) Cortical sources of the early components of the visual evoked potential. Hum Brain Mapp, 15, 95-111.

Eriksen, C. W., \& Yeh, Y. Y. (1985) Allocation of attention in the visual field. [Research Support, U.S. Gov't, P.H.S.]. J Exp Psychol, 11, 583-597. 
Estrada, C. A., Isen, A. M., \& Young, M. J. (1997) Positive affect facilitates integration of information and decreases anchoring in reasoning among physicians. Organ Behav Hum Dec, 72, 117-135.

Finucane, A. M., Whiteman, M. C., \& Power, M. J. (2010) The effect of happiness and sadness on alerting, orienting, and executive attention. J Atten Disord, 13, 629-639.

Fredrickson, B. (2001) The role of positive emotions in positive psychology: The broadenand-build theory of positive emotions. Am Psychol, 56, 218-226.

Fredrickson, B. (2004) The broaden-and-build theory of positive emotions. Philos Trans $R$ Soc Lond B, 359, 1367-1377.

Fredrickson, B., \& Branigan, C. (2005) Positive emotions broaden the scope of attention and thought-action repertoires. Cognition Emotion, 19, 313-332.

Fuchs, M., Kastner, J., Wagner, M., Hawes, S., \& Ebersole, J. S. (2002) A standardized boundary element method volume conductor model. Clinical Neurophysiology, 113, $702-712$.

Gasper, K., \& Clore, G. L. (2002) Attending to the big picture: Mood and global versus local processing of visual information. Psy Sci, 13, 34-40.

Gratton, G., Coles, M. G. H., \& Donchin, E. (1983) A New Method for Off-Line Removal of Ocular Artifact. Electroencephalogr Clin Neurophysiol 55, 468-484.

Gschwind, M., Pourtois, G., Schwartz, S., Van De Ville, D., \& Vuilleumier, P. (2012) Whitematter connectivity between face-responsive regions in the human brain. Cereb Cortex, 22, 1564-1576.

Handy, T. C., Soltani, M., \& Mangun, G. R. (2001) Perceptual load and visuocortical processing: Event-related potentials reveal sensory-level selection. Psy Sci, 12, 213218. 
Heinze, H. J., Luck, S. J., Münte, T. F., Gös, A., Mangun, G. R., \& Hillyard, S. A. (1994) Attention to adjacent and seperate positions in space: An electrophysiological analysis. Percept Psychophys, 56, 45-52.

Heinze, H. J., \& Mangun, G. R. (1995) Electrophysiological Signs of Sustained and Transient Attention to Spatial Locations. Neuropsychologia, 33, 889-908.

Heinze, H. J., Mangun, G. R., Burchert, W., Hinrichs, H., Scholz, M., Munte, T. F., et al. (1994) Combined spatial and temporal imaging of brain activity during visual selective attention in humans. Nature, 372, 543-546.

Hillyard, S. A., \& Anllo-Vento, L. (1998) Event-related brain potentials in the study of visual selective attention. Proc Natl Acad Sci U S A, 95, 781-787.

Holmes, E. A. (2006) Positive interpretation training: Effects of mental imagery versus verbal training on positive mood. Behav ther, 37, 237-247.

Holmes, E. A., Coughtrey, A. E., \& Connor, A. (2008) Looking at or Through Rose-Tinted Glasses? Imagery Perspective and Positive Mood. Emotion, 8, 875-879.

Huntsinger, J. R., Clore, G. L., \& Bar-Anan, Y. (2010) Mood and Global-Local Focus:

Priming a Local Focus Reverses the Link Between Mood and Global-Local Processing. Emotion, 10, 722-726.

Isen, A. M. (2000) Positive affect and decision making. In: Lewis, M. \& Haviland-Jones, J. M. (Eds). Handbook of Emotions (pp. 417-435). New York: Guilford Press.

Isen, A. M., \& Daubman, K. A. (1984) The Influence of Affect on Categorization. J Pers Soc Psychol, 47, 1206-1217.

Isen, A. M., Daubman, K. A., \& Nowicki, G. P. (1987) Positive Affect Facilitates Creative Problem-Solving. Journal of personality and social psychology, 52, 1122-1131.

Isen, A. M., Rosenzweig, A. S., \& Young, M. J. (1991) The Influence of Positive Affect on Clinical Problem-Solving. Med Decis Making, 11, 221-227. 
Ivry, R. B., \& Robertson, L. C. (1998) The two sides of perception. Cambridge, MA: The MIT Press.

Jans, B., Peters, J. C., \& De Weerd, P. (2010) Visual spatial attention to multiple locations at once: The jury is still out. Psychol Rev, 117, 637-684.

Jeffreys, D. A., \& Axford, J. G. (1972) Source Locations of Pattern-Specific Components of Human Visual Evoked-Potentials .1. Component of Striate Cortical Origin. Exp Brain Res, 16, 1-21.

Jurcak, V., Tsuzuki, D., \& Dan, I. (2007) 10/20, 10/10, and 10/5 systems revisited: Their validity as relative head-surface-based positioning systems. Neuroimage, 34, 16001611.

Kelly, S. P., Gomez-Ramirez, M., \& Foxe, J. J. (2008) Spatial attention modulates initial afferent activity in human primary visual cortex. Cereb Cortex, 18, 2629-2636.

Kim, K. H., Kim, J. H., Yoon, J., \& Jung, K. Y. (2008) Influence of task difficulty on the features of event-related potential during visual oddball task. Neurosci Lett, 445, 179183.

Kok, A. (2001) On the utility of P3 amplitude as a measure of processing capacity. Psychophysiology 38, 557-577.

Lakens, D. (2013) Calculating and reporting effect sizes to facilitate cumulative science: a practical primer for -tests and ANOVAs. Front Psychol, 4, 863.

Luck, S. J., Woodman, G. F., \& Vogel, E. K. (2000) ERP studies of attention. Trends cogn sci, 4, 432-440.

Mangun, G. R., Buonocore, M. H., Girelli, M., \& Jha, A. P. (1998) ERP and fMRI measures of visual spatial selective attention. Hum Brain Mapp, 6, 383-389.

Martin, E. A., \& Kerns, J. G. (2011) The influence of positive mood on different aspects of cognitive control. Cognition Emotion, 25, 265-279. 
Martínez, A., Anllo-Vento, L., Sereno, M. I., Frank, L. R., Buxton, R. B., Dubowitz, D. J., et al. (1999) Involvement of striate and extrastriate visual cortical areas in spatial attention. Nat Neurosci, 2, 364-369.

Mazziotta, J., Toga, A., Evans, A., Fox, P., Lancaster, J., Zilles, K., et al. (2001) A probabilistic atlas and reference system for the human brain: International Consortium for Brain Mapping (ICBM). Philos Trans R Soc Lond B Biol Sci, 356, 1293-1322.

McCarthy, G., \& Donchin, E. (1981) A metric for thought: a comparison of P300 latency and reaction time. Science, $211,77-80$.

Mitterschiffthaler, M. T., Fu, C. H. Y., Dalton, J. A., Andrew, C. M., \& Williams, S. C. R. (2007) A functional MRI study of happy and sad affective states induced by classical music. Human Brain Mapping, 28, 1150-1162.

Moors, A., \& De Houwer, J. (2006) Automaticity: A theoretical and conceptual analysis. Psychol Bull, 132, 297-326.

Moriya, H., \& Nittono, H. (2011) Effect of mood states on the breadth of spatial attentional focus: An event-related potential study. Neuropsychologia, 49, 1162-1170.

Muller, N. G., Bartelt, O. A., Donner, T. H., Villringer, A., \& Brandt, S. A. (2003) A physiological correlate of the "zoom lens" of visual attention. J Neurosci, 23, 35613565 .

Nieuwenhuis, S., Aston-Jones, G., \& Cohen, J. D. (2005) Decision making, the P3, and the locus coeruleus-norepinephrine system. [Research Support, Non-U.S. Gov't Review]. Psychol Bull, 131, 510-532.

Pascual-Marqui, R. D. (2002) Standardized low-resolution brain electromagnetic tomography (sLORETA): Technical details. Methods Find Exp Clin Pharmacol, 24, 5-12.

Pessoa, L. (2008) On the relationship between emotion and cognition. Nature Rev Neurosci, 9, 148-158. 
Picton, T. W., Bentin, S., Berg, P., Donchin, E., Hillyard, S. A., Johnson, R., et al. (2000) Guidelines for using human ERP to study cognition: Recognition standards and publication data. Psychophysiology, 37, 127-152.

Polich, J. (2007) Updating p300: An integrative theory of P3a and P3b. Clin Neurophysiol, $118,2128-2148$

Polich, J., \& Kok, A. (1995) Cognitive and biological determinants of P300: an integrative review. Biol Psychol, 41, 103-146.

Portzky, M., Wagnild, G., De Bacquer, D., \& Audenaert, K. (2010) Psychometric evaluation of the Dutch Resilience Scale RS-nl on 3265 healthy participants: a confirmation of the association between age and resilience found with the Swedish version. Scand $J$ Caring Sci, 24, 86-92.

Pourtois, G., Grandjean, D., Sander, D., \& Vuilleumier, P. (2004) Electrophysiological correlates of rapid spatial orienting towards fearful faces. [Clinical Trial Research Support, Non-U.S. Gov't]. Cereb cortex, 14, 619-633.

Pourtois, G., Schettino, A., \& Vuilleumier, P. (2013) Brain mechanisms for emotional influences on perception and attention: what is magic and what is not. Biol Psychol, $92,492-512$.

Rauss, K. S., Pourtois, G., Vuilleumier, P., \& Schwartz, S. (2009) Attentional load modifies early activity in human primary visual cortex. Hum Brain Mapp, 30, 1723-1733.

Rauss, K. S., Schwartz, S., \& Pourtois, G. (2011) Top-down effects on early visual processing in humans: A predictive coding framework. Neurosci Biobehav Rev, 35, 1237-1253.

Rossi, V., \& Pourtois, G. (2012) State-dependent attention modulation of human primary visual cortex: a high density ERP study. Neuroimage, 60, 2365-2378.

Rossi, V., \& Pourtois, G. (2013) Negative affective state mimics effects of perceptual load on spatial perception. Emotion, 13, 485-496. 
Rowe, G., Hirsh, J. B., \& Anderson, A. K. (2007) Positive affect increases the breadth of attentional selection. Proc Natl Acad Sci U S A, 104, 383-388.

Sawaki, R., \& Katayama, J. (2007) Difficulty of discrimination modulates attentional capture for deviant information. Psychophysiology, 44, 374-382.

Schwartz, S., Vuillemier, P., Hutton, C., Maravita, A., Dolan, R. J., \& Driver, J. (2005) Attentional load and sensory competition in human vision: Modulation of fMRI responses by load at fixation during task-irrelevant stimulation in the peripheral visual field. Cereb cortex, 15, 770-786.

Srinivasan, N., \& Hanif, A. (2010) Global-happy and local-sad: Perceptual processing affects emotion identification. Cognition Emotion, 24, 1062-1069.

Stolarova, M., Keil, A., \& Moratti, S. (2006) Modulation of the C1 visual event-related component by conditioned stimuli: evidence for sensory plasticity in early affective perception. Cereb Cortex, 16, 876-887.

Vanlessen, N., Rossi, V., De Raedt, R., \& Pourtois, G. (2013) Positive emotion broadens attention focus through decreased position-specific spatial encoding in early visual cortex: evidence from ERPs. Cogn Affect Behav Neurosci, 13, 60-79.

Vogel, E. K., \& Luck, S. J. (2000) The visual N1 component as an index of a discrimination process. Psychophysiology, 37, 190-203.

Vuilleumier, P. (2005) How brains beware: neural mechanisms of emotional attention. Trends Cogn Sci, 9, 585-594.

Watson, D., Clark, L. A., \& Tellegen, A. (1988) Development and validation of brief measures of positive and negative affect: the PANAS scales. J Pers Soc Psychol, 54, 1063-1070. 
Weymar, M., Keil, A., \& Hamm, A. O. (2013) Timing the fearful brain: unspecific hypervigilance and spatial attention in early visual perception. Soc Cogn Affect Neurosci.

\section{FIGURES}

Figure 1. Stimuli and task. A) Participants performed a dual task consisting of a demanding oddball task at fixation and a localization task (with peripheral vision) of textures shown at three concurrent spatial positions in the upper visual field. Stimuli at fixation and peripheral textures never overlapped in space nor time. B) Visual textures at the three possible positions in the upper visual field: Close, Middle or Far relative to fixation. Only the middle position was task-relevant and required an overt response. C) Half of the peripheral textures were made up by snowflake-like elements, while the other half consisted of 'crosses', with an equal number of presentations of each type at each of the three locations. Texture content was taskirrelevant.

Figure 2. Mood scores at baseline and after the MIP (average of the different measurements following the first MIP) of A) the VAS for feelings of happiness and B) feelings of pleasantness, and C) the positive affect scale of the PANAS. VAS scores for happiness and pleasantness increased after the MIP in the positive mood group, while they remained unchanged after the MIP (relative to the baseline) in the neutral mood group. The positive affect measured with the PANAS decreased during the experiment in the neutral mood group, but remained stable in the positive mood group. $* *$ indicates a significant effect with $p \leq .001$ and $* x \leq .05$; error bars represent 1 S.E.M.

Figure 3. A) Grand average ERPs to central stimuli (primary task) recorded at a representative posterior parietal midline electrode position (A20). A clear increase of the P300 component was found for oddball target stimuli (dashed line) compared to standard stimuli 
(solid line). However, the target P300 was larger in the positive (red) compared to the neutral (blue) mood group. No group difference was found for the P300 in response to the standard stimuli. The P300 was scored as the mean ERP activity recorded during a prolonged time interval (spanning from 490 to $690 \mathrm{~ms}$ post-stimulus onset; demarked by the grey frame). Note that negative values are plotted upwards. B) Corresponding voltage maps (back view) for the P300 component for the standard and target stimuli, separately for the positive and the neutral mood group.

Figure 4. A) Grand average ERPs to peripheral textures shown in the upper visual field (secondary task) recorded at a representative posterior parietal midline electrode position (A21/POz), separately for the three positions. While, as expected, the amplitude of the C1 monotonically decreased with increasing eccentricity relative to fixation, it was systematically larger in the positive (red) compared to the neutral (blue) mood group. Note that only the Middle position was task-related. The $\mathrm{C} 1$ was scored in individual averages as the mean ERP activity recorded during a $20 \mathrm{~ms}$ interval around the peak (demarked by the grey frame). Negative values are plotted upwards. B) Across the three positions, the C1 was larger in the positive (red) compared to the neutral mood group (blue). * indicates a significant effect with $p \leq .05$; error bars represent 1 S.E.M. C) Corresponding voltage maps for the C1, separately for each stimulus position and each group. D) Direct statistical comparison in the inverse solution space (sLoreta) between the positive mood group $(n=20)$ and the neutral mood group $(\mathrm{n}=20)$ for the $\mathrm{C} 1$ (peak amplitude) generated in response to the peripheral textures (all three positions aggregated). This analysis revealed a significantly higher activation in the positive compared to the neutral mood group encompassing early visual areas (Brodmann areas 17 and 18). The maximum activation $(t(39)=3,44, p<.005)$ was found in $\mathrm{x}=-5, \mathrm{y}=-80$, $\mathrm{Z}=10$ (MNI coordinates), corresponding to the vicinity of the calcarine fissure. This statistical 
analysis is based on a stringent non-parametric randomization test (relying on 5000 iterations), and provides corrected p-values.

Figure 5. Grand average ERPs to peripheral stimuli (Close position) recorded at a representative posterior parietal midline electrode position (A21/POz), separately for textures shown in the upper (black line) and in the lower visual field (grey line) (localizer blocks); and for A) the positive and B) the neutral mood group (Localizer). In each group, a conspicuous polarity reversal was evidenced for the $\mathrm{C} 1$ as a function of the position of the stimulus in the visual field (i.e., upper visual field presentations were associated with a negative deflection while lower visual field presentations were associated with a positive deflection at the same latency). The corresponding voltage maps are shown. C) For the P1 (main experimental session; secondary task), the analysis showed a reduced amplitude for the Far position, relative to the two other positions, without a modulation by (positive) mood. The corresponding voltage maps are shown for a time window of 105-125 ms post-stimulus onset. D) For the N1 (main experimental session; secondary task), the analysis showed that the texture made up of snowflakes (Texture A) elicited a larger N1 (the voltage maps are shown for an interval of 105-125 ms post-stimulus onset), than the texture made up of crosses (Texture B), equally so in each group (positive/red and neutral/blue). ** indicates a significant effect with $p \leq .005$ and $* p \leq .01$; error bars represent 1 S.E.M. 


\begin{tabular}{llccccc}
\hline VAS & Group & Baseline & MIP1 & MIP2 & MIP3 & $\begin{array}{c}\text { After last } \\
\text { block }\end{array}$ \\
\hline \multirow{4}{*}{ Happiness } & Positive & 5.42 & 8.01 & 7.66 & 7.95 & 5.54 \\
& & $(2.72)$ & $(1.77)$ & $(1.94)$ & $(1.82)$ & $(2.69)$ \\
\cline { 2 - 7 } & Neutral & 4.51 & 4.23 & 4.20 & 4.07 & 3.87 \\
& & $(2.84)$ & $(2.78)$ & $(2.79)$ & $(2.88)$ & $(2.89)$ \\
\hline \multirow{4}{*}{ Sadness } & Positive & 0.61 & 0.54 & 0.42 & 0.53 & 0.51 \\
& & $(0.73)$ & $(0.89)$ & $(0.66)$ & $(0.95)$ & $(0.60)$ \\
\cline { 2 - 7 } & Neutral & 0.60 & 0.91 & 0.71 & 0.70 & 0.74 \\
& & $(0.79)$ & $(1.32)$ & $(0.76)$ & $(0.82)$ & $(1.45)$
\end{tabular}

Table 1. Mean VAS scores (+1 standard deviation) obtained for the feelings happiness and sadness at the different measurement points during the experiment, separately for the positive and the neutral mood group. These results show a steep increase of happiness following the first MIP in the positive mood group, exclusively. High levels of happiness were also maintained throughout the experiment in this group. By contrast, levels of sadness were low and balanced between the two groups. 


\begin{tabular}{|ll|c|c|c|}
\cline { 3 - 5 } \multicolumn{1}{c|}{} & \multicolumn{2}{c|}{ Vanlessen et. al, 2013 } & Current study \\
\hline EEG measurement & & Exp 1 & Exp 2 & \\
\hline Primary task & & yes & no & yes \\
Behavior & ACC & $=$ & Detection & Detection \\
& RT & N/A & $=$ & $=$ \\
ERP & P300 & $=$ & $=$ & Numerically faster \\
\hline Secondary task & & NA & Discrimination & Localization \\
Behavior & ACC & N/A & Lower & $=$ \\
& RT & N/A & $=$ & Faster \\
ERP & C1 & Larger & N/A & Larger (amplitude) \\
& P1 & (topography) & N/A & $=$ \\
& N1 & N/A & N/A & $=$ \\
\hline
\end{tabular}

Table 2. Systematic comparison between the results obtained in our previous ERP study (Vanlessen et al., 2013) and the current results. These symbols (and adjectives) reflect the direction of the effect found when comparing the positive mood group to the neutral mood group. For example, "larger" means that the positive group showed a larger component as compared to the neural group. Note that this systematic comparison between these two studies is made difficult because of reliable methodological differences between them. In Vanlessen et.al, 2013, no (secondary) task with the peripheral textures was required while EEG was recorded concurrently (Experiment 1). In a control experiment without EEG (Experiment 2), participants were instructed to discriminate the content of these peripheral stimuli, regardless of their location ("what" task), besides the primary task. In the current study, participants had to localize these peripheral stimuli, regardless of their content ("where" task). Despite these methodological differences across the two studies, we found that the amplitude of the $\mathrm{C} 1$ to the peripheral textures was augmented (either in topography in Vanlessen et al., 2013 or amplitude in the present case) in the positive compared to the neutral mood group. N/A means Not Applicable. 
(A)

Central stimulus (150 ms)

(250 ms)

$+$

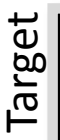

.
$+$
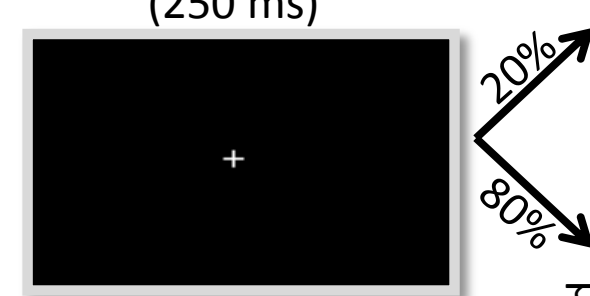

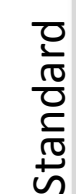

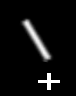

$+$
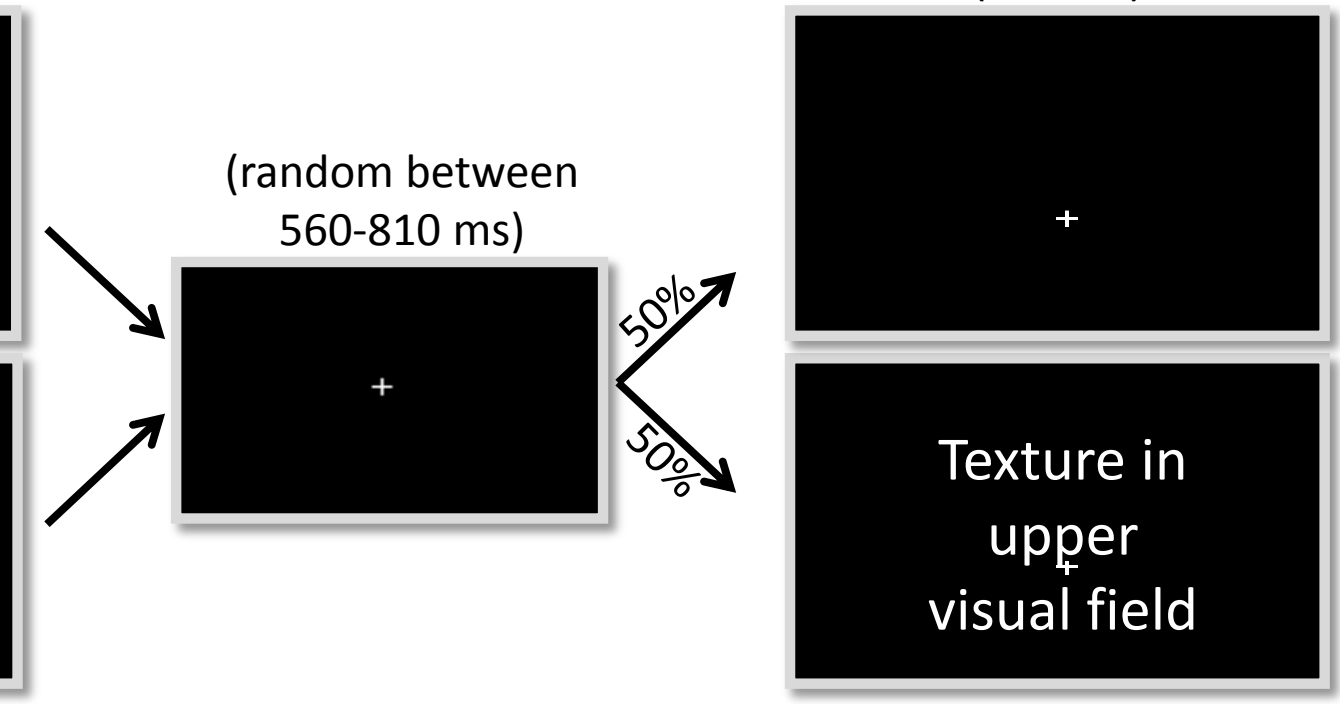

\section{Texture in} upper visual field

(B)
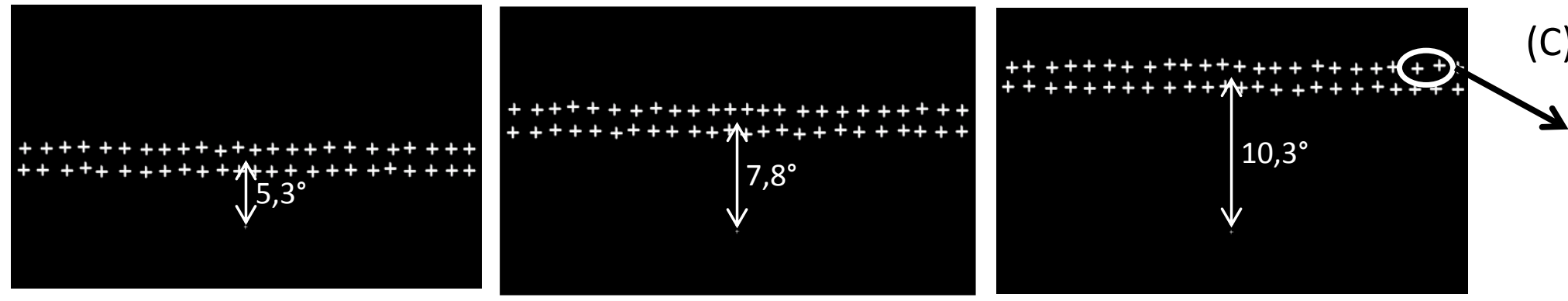

金忩 $+t$ 
(A)

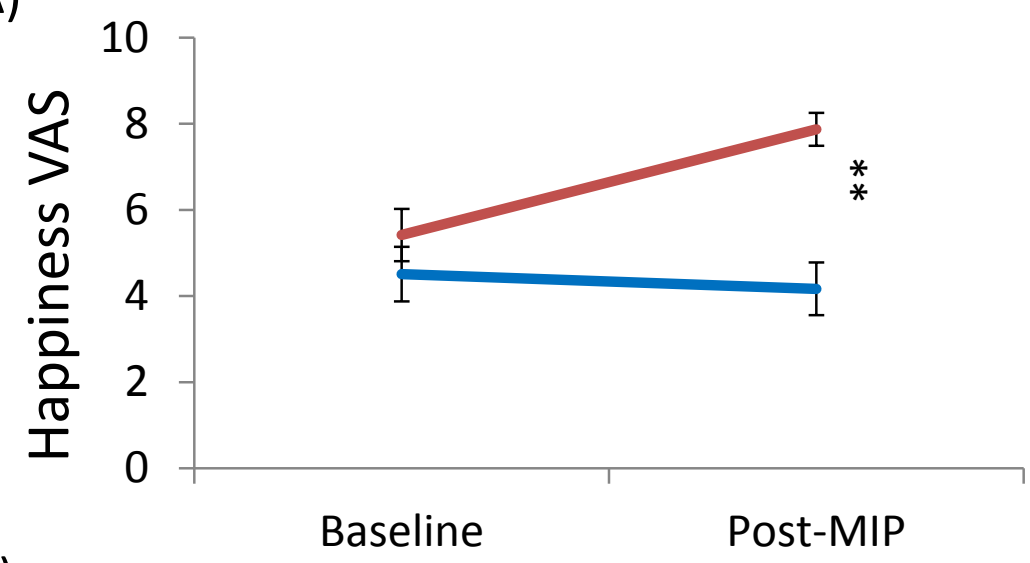

(B)

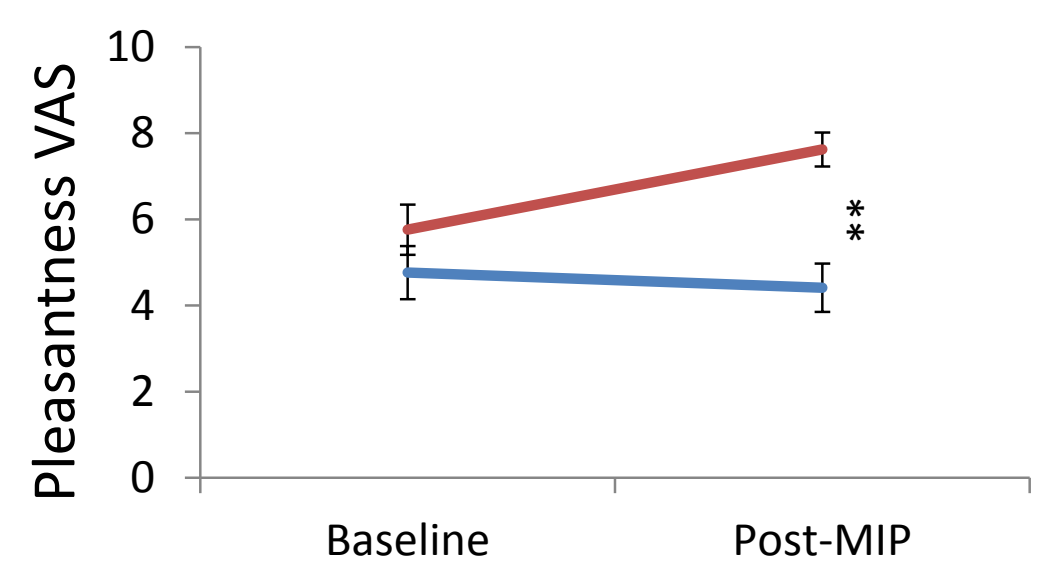

(C)

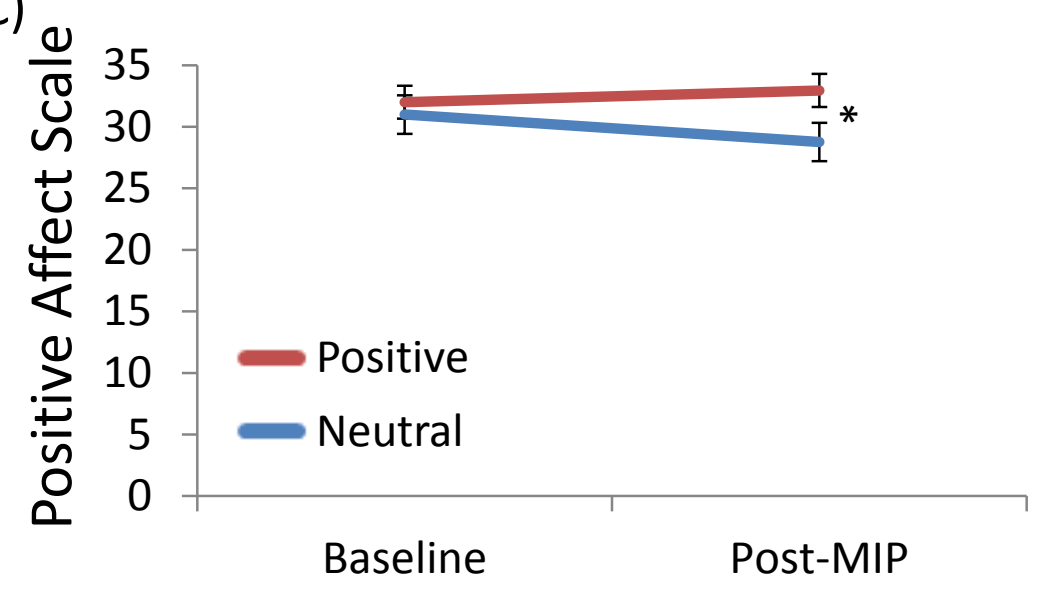



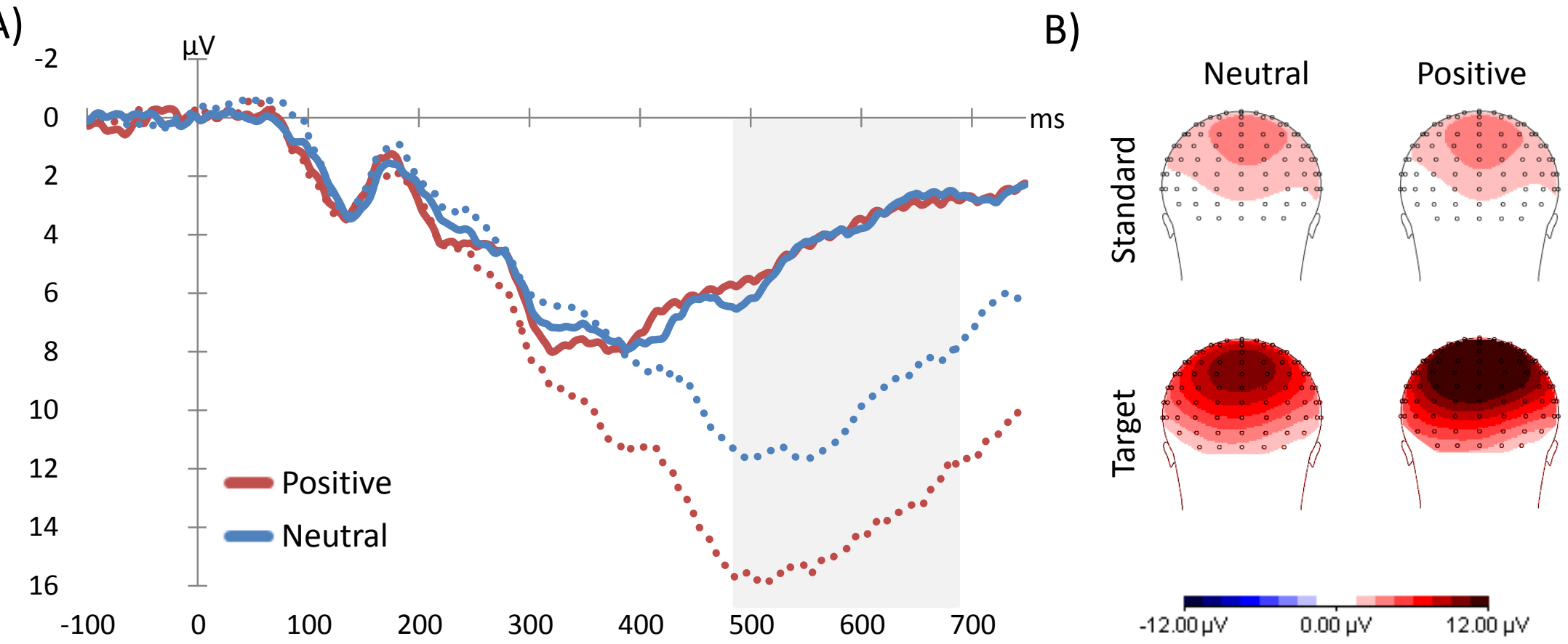

B) 
